\title{
El pH de la carne de cobayo (Cavia porcellus) para consumo humano en los andes centrales del Perú
}

\author{
Lucas, J.R. ${ }^{1}$; Balcázar, S. ; Tirado, O. ${ }^{1}$; Rodríguez, A. ${ }^{2}$ \\ ${ }^{1}$ Centro de Investigaciones IVITA, Estación El Mantaro, Facultad de Medicina Veterinaria, Universidad \\ Nacional Mayor de San Marcos, Junín, Perú. ${ }^{2}$ Servicio Nacional de Sanidad Agraria, SENASA, Junín, Perú. \\ E-mail: rlucas.pe@gmail.com
}

\begin{abstract}
Resumen
Lucas, J.R.; Balcázar, S.; Tirado, O.; Rodríguez, A.: El pH de la carne de cobayo (Cavia porcellus) para consumo humano en los andes centrales del Perú. Rev. vet. 29: 1, 65-67, 2018. El objetivo del presente estudio fue evaluar el $\mathrm{pH}$ de la carne de cobayos faenados en los andes centrales del Perú. Se determinó el $\mathrm{pH}$ de 60 canales al terminar el proceso de sacrificio y a la $1,6,12$, y $24 \mathrm{~h}$ posteriores. Los $\mathrm{pH}$ fueron de $6,79 \pm 0,3,6,74 \pm 0,34,6,59 \pm$ $0,28,6,45 \pm 0,35$ y $5,96 \pm 0,11$, a las horas $0,1,6,12$ y 24 post sacrificio respectivamente. La ecuación que explica el comportamiento del $\mathrm{pH}$ fue $\mathrm{Y}=6.7765-0.0231 \mathrm{X}-0.0005 \mathrm{X}^{2}\left(\mathrm{R}^{2}=0.996\right)$. La ausencia de un adecuado descenso del $\mathrm{pH}$ muscular incide negativamente en la calidad de la carne debido a la proliferación de microrganismos perjudiciales. Para mejorar la calidad y prolongar la vida útil de la carne de cobayo se requieren estudios que evalúen las repercusiones de las deficiencias constatadas durante el sacrificio, dado que no se observó descanso, ducha ante mortem ni aturdimiento.
\end{abstract}

Palabras clave: Cavia porcellus, carne para consumo, sacrificio, reducción del pH, fallas detectadas.

\begin{abstract}
Lucas, J.R.; Balcázar, S.; Tirado, O.; Rodríguez, A.: The pH of meat of guinea pig (Cavia porcellus) for human consumption in the peruvian central Andes. Rev. vet. 29: 1, 6567, 2018. The aim of this study was to evaluate the $\mathrm{pH}$ of meat of Guinea pig from peruvian central andes. The $\mathrm{pH}$ of 60 guinea pig carcasses were analyzed at the end of the slaughter process and at 1, 6, 12 and $24 \mathrm{~h}$ later. The $\mathrm{pH}$ was $6.79 \pm 0.3,6.74 \pm 0.34,6.59 \pm 0.28,6.45 \pm$ 0.35 and $5.96 \pm 0.11$ for $0,1,6,12$ y $24 \mathrm{~h}$ post slaughtering. The equation that graphs $\mathrm{pH}$ was $\mathrm{Y}=6.7765-0.0231 \mathrm{X}-0.0005 \mathrm{X}^{2}\left(\mathrm{R}^{2}=0.996\right)$. The absence of an appropriate descent of the muscular $\mathrm{pH}$ impacts negatively in the meat quality due to the proliferation of harmful bacteria. Studies are required to assess the impact of the deficiencies observed during slaughtering (it was not observed lairage nor ante-mortem shower and guinea pigs were not stunned prior to bleeding and sticking was carried out with conscious animals) on the quality and shelf life of guinea pig meat.
\end{abstract}

Key words: Cavia porcellus, meat consumption, sacrifice, $\mathrm{pH}$ reduction, detected failures.

\section{INTRODUCCIÓN}

Perú es el país con la mayor población de cobayos o "cuyes" (Cavia porcellus) en el mundo, produciendo 16.500 tn de carne proveniente del sacrificio de aproximadamente 65 millones de estos animales, para consumo humano. El "cuy" es un animal que por su capacidad de adaptación está distribuido en todo el Perú, aunque es especialmente importante en los andes, donde se encuentra en mayor cantidad y es una fuente proteica muy popular ${ }^{4}$.

El pH del músculo es de 7,0-7,6. Después del sacrificio, durante su transformación en carne, el músculo obtiene ATP para su relajación a través de la vía anaeróbica de la glicólisis. Esto resulta en la acumulación de lactato e hidrogeniones, disminuyendo el $\mathrm{pH}$ de la carne ${ }^{5,7}$. Tal descenso inhibe la velocidad de crecimiento de los microorganismos patógenos y de los que alteran la carne, pues éstos crecen óptimos alrededor de $\mathrm{pH} 7$ o ligeramente alcalino ${ }^{2}$.

El manejo inadecuado del animal durante el sacrificio influye negativamente sobre el descenso del $\mathrm{pH}$ en la carne $9,12,15,18$. En la sierra central del Perú es común el sacrificio del cobayo con deficiencias que atentan contra el bienestar animal, por lo cual el objetivo del presente trabajo fue evaluar el $\mathrm{pH}$ de la carne resultante de estos procesos. 


\section{MATERIAL Y MÉTODOS}

Lugar de estudio y animales. El trabajo fue realizado en un matadero autorizado de Jauja (Junín, Perú, a $3.000 \mathrm{msnm}$ ), entre noviembre-diciembre de 2013 y marzo-abril de 2014. Se evaluó el pH de 60 canales de cobayos machos de la línea $\mathrm{G}^{10}$, de entre $900-1.000 \mathrm{~g}$ de peso vivo, 1,5-2,0 meses de edad, criados en forma tecnificada y alimentados con una combinación de forraje $(80 \%)$ y concentrado $(20 \%)$.

El sacrificio en el matadero constó de las siguientes etapas: degüello y desangrado, escaldado $\left(60-70^{\circ} \mathrm{C}\right.$ por 30-60 seg), eviscerado (mediante un corte longitudinal desde la sínfisis púbica hasta el corte del degüello), retoque, lavado y enfriado de la canal en agua (aproximadamente $4-6^{\circ} \mathrm{C}$ ) y con hipoclorito de sodio. Finalmente, las canales tuvieron un periodo de escurrido en la sala de oreo, entre 40-60 min.

Ningún animal recibió descanso ni ducha ante mortem. Tampoco se realizó la aplicación del aturdimiento y en todos los casos los animales fueron sacrificados por degüello directo. Como requerimiento del comercio local, las canales incluían los pulmones, hígado, corazón, riñones, testículos y la cabeza.

Diseño experimental. Se evaluó el $\mathrm{pH}$ en la sala de oreo del matadero al momento de finalizado el proceso de sacrificio (T0) y a las 6,12 y 24 horas posteriores. Además, se midió el $\mathrm{pH}$ a los $60 \mathrm{~min}$ de finalizado el proceso, por ser el tiempo de espera más frecuente en el que permanecen las canales en la sala de oreo antes de ser distribuidas al mercado.

El tamaño de muestra mínimo $(\mathrm{n}=10)$ fue obtenido con la fórmula para estimación de una media en poblaciones infinitas, con una confianza del $90 \%$, un error máximo admisible del $10 \%$ y una desviación estándar esperada del 0,1. Por disponibilidad, se evaluaron 60 canales en 4 procesos de sacrificio.

$\mathrm{El} \mathrm{pH}$ fue medido en el músculo Longissimus dorsi de la porción lumbar, para ello se efectuó un corte de $15-20 \mathrm{~mm}$ de profundidad, incidiendo la piel y el músculo. La lectura del $\mathrm{pH}$ se efectuó con potenciómetro digital portátil (Hanna, HI 8424), dotado de un electrodo de inserción para alimentos, con una resolución de 0,01 unidades de $\mathrm{pH}$.

Análisis estadístico. Se realizó un modelo de regresión cuadrática con el $\mathrm{pH}$, utilizando el paquete estadístico SPSS. Se determinó el coeficiente de regresión, así como el tipo de función que mejor se ajusta a las observaciones de $\mathrm{pH}: \mathrm{Y}=\beta 0-\beta 1 \mathrm{X}+\beta 2 \mathrm{X}^{2}$, donde: $\mathrm{Y}$ $=$ representa el valor del $\mathrm{pH}, \mathrm{X}=$ representa el tiempo post-faenamiento en horas y $\beta(1,2)=$ las constantes.

\section{RESULTADOS Y DISCUSIÓN}

$\mathrm{El} \mathrm{pH}$ al final del proceso de sacrificio (T0) fue de $6,79 \pm 0,3$. Asimismo, el $\mathrm{pH}$ fue de $6,74 \pm 0,34,6,59 \pm$ $0,28,6,45 \pm 0,35$ y $5,96 \pm 0,11$, a la $1,6,12$ y 24 horas

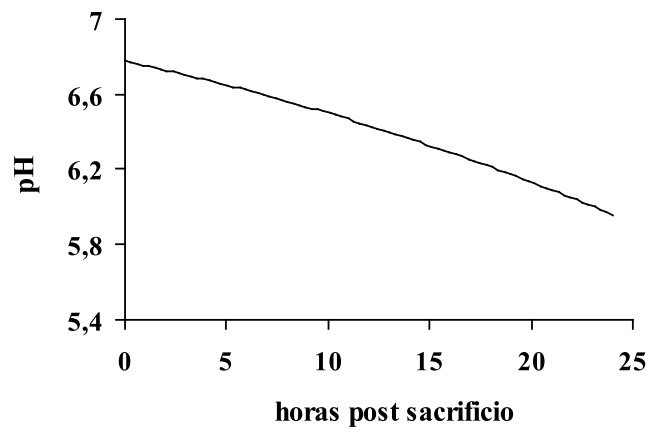

Figura 1. Evolución del pH de la carne tras 24 horas de sacrificio.

post sacrificio respectivamente. La ecuación que grafica mejor el comportamiento de este parámetro fue: $\mathrm{Y}=6.7765-0.0231 \mathrm{X}-0.0005 \mathrm{X}^{2}\left(\mathrm{R}^{2}=0.996\right)$. La Figura 1 muestra la evolución del $\mathrm{pH}$.

$\mathrm{El} \mathrm{pH}$ a las $24 \mathrm{~h}$ post sacrificio ( $\mathrm{pH} 24 \mathrm{~h}$ ) es un indicador de calidad que se usa para valorar la caída del $\mathrm{pH}$. El valor del pH 24 h es específico para cada especie y además está influenciado por muchos factores como la edad, el sexo y la procedencia. En bovinos un pH 24 $\mathrm{h} \geq 5,8$ resulta inadecuado $\mathrm{y}$, consecuentemente, podría suponer una carne con menor vida útil y con características sensoriales inapropiadas ${ }^{3}$.

Se ha reportado un pH 24 h de 6,06 en los músculos psoas de cobayos machos explotados a nivel del mar, aturdidos y descansados ${ }^{14}$, es decir un resultado similar al aquí obtenido.

En el presente trabajo se observaron prácticas que atentan contra el bienestar animal y que podrían estar generando un estrés importante en el cobayo durante el sacrifico, como la falta de descanso ante mortem y el aturdimiento previo. Se sabe que el estrés ante mortem disminuye las reservas de glucógeno en los animales de abasto lo cual, posteriormente, afectará las características fisicoquímicas de la carne, incluido el descenso del $\mathrm{pH}^{3,9,12,15,18}$.

El correcto aturdimiento produce inconsciencia y, por ende, un estrés insignificante en el animal. Sin embargo, un método de aturdimiento incorrecto resulta más estresante que el sacrificio sin aturdimiento ${ }^{11}$. Asimismo, en el comercio local la canal del cobayo se expende con la cabeza y el hecho de aplicar algunos métodos vigentes, como la conmoción por perno cautivo o el shock eléctrico (que coloca conductores de electricidad a nivel de la cabeza), podrían causar rechazo en el consumidor, pues ambos producen marcas visibles en la cabeza de la canal.

Por otro lado, la Food \& Agriculture Organization (FAO, United Nations) indica que el descanso ante mortem no debe superar las $72 \mathrm{~h}$, siendo común en la industria aplicar entre 6 y $12 \mathrm{~h}^{6}$. Durante dicho descanso los animales sufren una ayuna que permite el vaciamiento parcial del tracto gastrointestinal (importante para un correcto eviscerado) y una recuperación de los niveles normales de glucógeno muscular ${ }^{7}$. 
La falta de descanso ante mortem, como la observada en los animales sacrificados en el presente estudio, predispone a la disminución de las reservas de glucógeno muscular. Contrariamente, un descanso extenso tiene repercusiones negativas sobre el estrés del animal.

El tiempo de descanso ante mortem está relacionado con la especie, el tiempo y las condiciones climáticas durante el transporte desde la granja al matadero ${ }^{8,17}$. Por tanto, son necesarios estudios que evalúen estos factores en la región andina. Se ha reportado que en la costa peruana el sacrificio cotidiano del cobayo consta de un descanso ante mortem de entre $10 \mathrm{~h}{ }^{16} \mathrm{y}$ $24 \mathrm{~h}^{14}$.

El requerimiento comercial de incluir las vísceras y la cabeza en la presentación final de la canal del cobayo es un peligro contra la inocuidad del producto, puesto que se asocia con la presencia de patógenos en la carne, como Salmonella sp, Campylobacter sp y Listeria monocytogenes, de forma similar a lo descrito previamente en pollos y cerdos en Lima, Perú ${ }^{1,13,19}$. Ello implica que también sería necesario evaluar las repercusiones de esta práctica.

Los pocos estudios que describen la evolución del $\mathrm{pH}$ de la carne de cobayo coinciden con nuestros resultados en indicar que el $\mathrm{pH} 24$ h es más elevado comparado con lo establecido en otros animales de abasto. Asimismo, se ha discutido sobre la repercusión de las prácticas de sacrificio observadas sobre la curva del $\mathrm{pH}$ obtenida, pero se requiere de estudios que evalúen el $\mathrm{pH}$ en ausencia de estas deficiencias, pudiendo utilizar la ecuación obtenida ( $\left.\mathrm{Y}=6.7765-0.0231 \mathrm{X}-0.0005 \mathrm{X}^{2} ; \mathrm{R}^{2}=0.996\right)$ como referencia para valoraciones posteriores.

\section{REFERENCIAS}

1. Barrientos E, Lucas JR, Ramos D, Rebatta M, Arbaisa T. 2015. Presencia de Listeria monocytogenes en canales porcinas en Lima, Perú. Rev Inv Vet Perú 26: 135-139.

2. Benito M. 2006. Higiene e inspección de carnes, $2^{\mathrm{a}}$ ed., Ediciones Díaz de Santos, Madrid, 646 p.

3. Brown S, Beavis E, Warris P. 1990. An estimate of the incidence of dark cutting beef in the United Kingdom. Meat Sci 27: 249-258.

4. Chauca LJ. 1997. Producción de cuyes (Cavia porcellus). Publicación de la Organización de las Naciones Unidas para la Agricultura y la Alimentación [FAO], Roma. http:// www.fao.org/docrep/w6562s/w6562s00.htm\#TopOfPage

5. De Fremery D. 1966. The physiology and biochemistry of muscle as food (Briskey EJ, Cassens RG, Trautmann JC, eds.), University of Wisconsin Press, Madison, USA, p 205.
6. FAO. Organización de las Naciones Unidas para la Agricultura y la Alimentación. 2007. Buenas prácticas para la industria de carne, Ed. FAO Roma, $44 \mathrm{p}$.

7. Forrest C, Aberle E, Hedrick H, Judge M, Merkel R. 1979. Fundamentos de la ciencia de la carne, Ed. Acribia, Zaragoza, $364 \mathrm{p}$.

8. Gallo C, Perez V, Sanhueza V. 2000. Efectos del tiempo de transporte de novillos previo al faenamiento sobre el comportamiento, las pérdidas de peso y algunas características de la canal. Arch Med Vet 32: 157-170.

9. Gallo C. 2009. Transporte y reposo pre-sacrificio en bovinos y su relación con la calidad de la carne. En: Bienestar animal y calidad de la carne (Mota D, Guerrero I, eds. ), BM Editores, México, p 15-36.

10. Jimenez R, Huaman A. 2010. Cuyes G, genéticamente geniales: Manual para el manejo de reproductores hibridos especializados en producción de carne, Ed. Universidad Nacional Mayor de San Marcos, Fac.Med.Vet., Huancayo, Perú, 175 p.

11. Kilgour R. 1978. The application of animal behavior and the humane care of farm animals. J Anim Sci 46: 1478-1486.

12. Kline K, Bechtel P. 1990. Effects of postmortem time and electrical stimulation on histochemical muscle fiber staining and $\mathrm{pH}$ in their middle gluteal muscle from beef cattle. Journal Food Quality 13: 447-452.

13. Lucas J, Vilca M, Ramos D. 2013. Presencia de Campylobacter spp en canales y ciegos de pollos de engorde en Lima, Perú. Rev Inv Vet Perú 24: 346-352.

14. Nakandakari L, Gutiérrez E, Chauca L, Valencia R. 2014. Medición del pH intramuscular del cuy (Cavia porcellus) durante las primeras 24 horas post beneficio tradicional. Salud Tecnol Vet 2: 99-105.

15. Sackmann G, Stolle F, Reuter G. 1989. Influencia de los diferentes tiempos de descanso previo al sacrificio sobre la calidad de la carne de cerdos con una evaluación de las características clínicas. Fleischwirtsch 1: 3-12.

16. Sánchez R, Silva M, Jiménez R, Zea O. 2015. Efecto de desinfectantes químicos y extractos de plantas sobre la carga bacteriana en carcasas de cuyes (Cavia porcellus). Rev Inv Vet Perú 26: 235-244.

17. Schaefer A, Jones S, Stanley R. 1997. The use of electrolyte solutions for reducing transport stress. J Anim Sci 75: 258-265.

18. Warriss P. 1990. The handling of cattle pre-slaughter and its effects on carcass and meat quality. Applied Anim Behav Sci 28: 171-186.

19. Zambrano H, Lucas JR, Vilca M, Ramos D. 2013. Determinación de Salmonella spp en centros de beneficio clandestino de pollos de engorde en Lima, Perú. Rev Inv Vet Perú 24: 337-345. 